\title{
The stability of an equation from mathematical finance without the boundary value condition
}

\section{Zhan Huashui $^{1 *}$}

\section{"Correspondence:}

huashuizhan@163.com

'School of Applied Mathematics,

Xiamen University of Technology,

Xiamen, China

\section{Springer}

\begin{abstract}
The strong degenerate parabolic equation

$$
\partial_{x x} u+u \partial_{y} u-\partial_{t} u=f(x, y, t, u)
$$

comes from the mathematics of finance. A kind of entropy solution is introduced. By Kružkov's bi-variable method, the stability for entropy solutions only depending on the initial value is proved. The usual boundary value condition is replaced by the regularity of the domain in a special sense.
\end{abstract}

MSC: 35L65; 35L85; 35R35; 62P05

Keywords: Mathematics finance; Entropy solution; Kružkov's bi-variables method; Boundary value condition

\section{Introduction}

Consider the strongly degenerate parabolic equation

$$
\partial_{x x} u+u \partial_{y} u-\partial_{t} u=f(x, y, t, u), \quad(x, y, t) \in Q_{T}=\Omega \times(0, T),
$$

where $\Omega \subset \mathbb{R}^{2}$ is a domain with the suitably smooth boundary $\partial \Omega$. Equation (1.1) arises from mathematical finance [1], or from nonlinear physical phenomena such as the combined effects of diffusion with convection of matter [2]. Antonelli, Barucci and Mancino [1] introduced a new model for an agent's decision under risk, in which the utility function is the solution of Eq. (1.1), in particular, $0 \leq u \leq 1$. The local classical solution of the Cauchy problem to Eq. (1.1) had been solved by Crandall, Ishii and Lions [3], and by Citti, Pascucci and Polidoro [4], Antonelli and Pascucci [5] step by step. However, Zhan [6] had shown that the global weak solution of Eq. (1.1) cannot be classical generally. In other words, some blow-up phenomena happen in finite time. Accordingly, we should concerned with the well-posedness of weak solutions generally.

If the weak solutions hold in the sense of entropy solutions, then the well-posedness as regards the Cauchy problem to Eq. (1.1) is included in our previous work [7, 8]. If Eq. (1.1)

(c) The Author(s) 2019. This article is distributed under the terms of the Creative Commons Attribution 4.0 International License (http://creativecommons.org/licenses/by/4.0/), which permits unrestricted use, distribution, and reproduction in any medium, provided you give appropriate credit to the original author(s) and the source, provide a link to the Creative Commons license, and indicate if changes were made. 
comes with the usual initial-boundary value conditions,

$$
\begin{aligned}
& u(x, y, 0)=u_{0}(x, y), \quad(x, y) \in \Omega, \\
& u(x, t)=0, \quad(x, t) \in \partial \Omega \times(0, T),
\end{aligned}
$$

when $f_{x}, f_{y}, f_{t}$ are bounded functions, and $f_{u}$ is bounded only if $u$ is bounded, then the existence and the uniqueness of the entropy solution is proved in [9]. The problem seemingly has been solved perfectly. But, since the equation lacks the term $\partial_{y y} u$, it is a strongly degenerate parabolic equation, the usual boundary value condition (1.3) might be superfluous. In fact, how to impose a suitable boundary value condition to match up with the following hyperbolic-parabolic mixed type equation:

$$
\frac{\partial u}{\partial t}=\sum_{i, j=1}^{N} \frac{\partial}{\partial x_{i}}\left(a^{i j}(x, t, u) \frac{\partial u}{\partial x_{j}}\right)+\operatorname{div}(b(x, t, u))+c(x, t, u), \quad(x, t) \in \Omega \times(0, T),
$$

has been an important problem in PDE for a long time. One may refer to Refs. [10-19] and the references therein. Here $\Omega \subset \mathbb{R}^{N}$ is a bounded domain.

In this paper, unlike [9-19], we avoid considering the boundary value condition tactfully. We primarily study whether the stability of entropy solutions of Eq. (1.1) with the initial value (1.2) can be proved without any boundary value condition directly. Unsurprisingly, we should try to find some other conditions to replace the boundary value condition.

Let us make some preparations. We say $u \in \operatorname{BV}\left(Q_{T}\right)$, if and only if $u \in L_{\text {loc }}^{1}\left(Q_{T}\right)$ and

$$
\int_{0}^{T} \int_{B_{\rho}}\left|u\left(x+h_{1}, y+h_{2}, t+h_{3}\right)-u(x, y, t)\right| d x d t \leq K|h|
$$

where

$$
B_{\rho}=\left\{X=(x, y) \in \mathbb{R}^{2}:|X|<\rho\right\}, \quad h=\left(h_{1}, h_{2}, h_{3}\right)
$$

and $K$ is a positive constant. Let $\Gamma_{u}$ be the set of all jump points of $u \in \operatorname{BV}\left(Q_{T}\right), v$ the normal of $\Gamma_{u}$ at $X=(x, t), u^{+}(X)$ and $u^{-}(X)$ the approximate limits of $u$ at $X \in \Gamma_{u}$ with respect to $(v, Y-X)>0$ and $(v, Y-X)<0$, respectively. For a continuous function $p$ : $(x, y, t, u) \rightarrow p(x, y, t, u)$ and $u \in \operatorname{BV}\left(Q_{T}\right)$, define

$$
\widehat{p}(x, y, t, u)=\int_{0}^{1} p\left(x, y, t, \tau u^{+}+(1-\tau) u^{-}\right) d \tau,
$$

which is called the composite mean value of $p$. Moreover, if $f \in C^{1}(\mathbb{R}), u \in \operatorname{BV}\left(Q_{T}\right)$, then $f(u) \in \operatorname{BV}\left(Q_{T}\right)$ and

$$
\frac{\partial f(u)}{\partial x_{i}}=\widehat{f}^{\prime}(u) \frac{\partial u}{\partial x_{i}}, \quad i=1,2, x_{1}=x, x_{2}=y
$$

is true in the sense of the weak derivatives, i.e. for any $\varphi(x, t) \in C_{0}^{1}\left(Q_{T}\right)$,

$$
\iint_{Q_{T}} \varphi(x, t) d\left[\frac{\partial f(u)}{\partial x_{i}}\right]=\iint_{Q_{T}} \widehat{f^{\prime}(u)} \varphi(x, t) d\left[\frac{\partial u}{\partial x_{i}}\right] .
$$


Let $S_{\eta}(s)=\int_{0}^{s} h_{\eta}(\tau) d \tau$ for small $\eta>0$. Here $h_{\eta}(s)=\frac{2}{\eta}\left(1-\frac{|s|}{\eta}\right)_{+}$, obviously $h_{\eta} \in C(\mathbb{R})$ and

$$
\begin{aligned}
& h_{\eta}(s) \geq 0, \quad\left|h_{\eta}(s)\right| \leq \frac{2}{\eta}, \quad\left|S_{\eta}(s)\right| \leq 1, \\
& \lim _{\eta \rightarrow 0} S_{\eta}(s)=\operatorname{sign}(s), \quad \lim _{\eta \rightarrow 0} s S_{\eta}^{\prime}(s)=0 .
\end{aligned}
$$

Definition 1.1 Let $\Omega$ be a bounded domain in $\mathbb{R}^{2}$. A function $u$ is said to be the entropy solution of Eq. (1.1) with the initial value (1.2), if:

(i) $u \in \operatorname{BV}\left(Q_{T}\right) \cap L^{\infty}\left(Q_{T}\right)$, and $u_{x} \in L^{2}\left(Q_{T}\right)$.

(ii) For any $0 \leq \varphi \in C_{0}^{2}\left(Q_{T}\right)$, for any $k \in \mathbb{R}$, for any $\eta>0$, $u$ satisfies

$$
\begin{aligned}
& \iint_{Q_{T}}\left[I_{\eta}(u-k) \varphi_{t}-B_{\eta}(u, k) \varphi_{y}+I_{\eta}(u-k) \varphi_{x x}\right. \\
& \left.\quad-f(\cdot, u) S_{\eta}(u-k) \varphi-S_{\eta}^{\prime}(u-k)\left(\partial_{x} u\right)^{2} \varphi\right] d x d y d t \\
& \quad \geq 0 .
\end{aligned}
$$

Here

$$
B_{\eta}(u, k)=\int_{k}^{u} s S_{\eta}(s-k) d s, \quad I_{\eta}(u-k)=\int_{0}^{u-k} S_{\eta}(s) d s .
$$

(iii) The initial value condition is true in the sense of that

$$
\lim _{t \rightarrow 0} \int_{\Omega}\left|u(x, y, t)-u_{0}(x, y)\right| d x d y=0
$$

The existence of an entropy solution has been given in [9], we do not repeat it here. The first aim of this paper is, by Kružkov's bi-variable method, to prove the stability of entropy solutions of Eq. (1.1) with the initial value (1.2), but without any boundary value condition.

Theorem 1.2 Let $\Omega$ be a bounded domain, $0 \leq u \leq 1,0 \leq v \leq 1$ be two solutions of Eq. (1.1) with the different initial values $u_{0}, v_{0} \in L^{\infty}(\Omega)$. Suppose that $f(\cdot, u)$ is a Lipschitz function, and that there exists a function $a \in C^{1}(\bar{\Omega}), a_{x x} \in C(\bar{\Omega})$,

$$
a(x, y)=0, \quad(x, y) \in \partial \Omega, \quad a(x, y)>0, \quad(x, y) \in \Omega
$$

such that when $(x, y)$ is near the boundary $\partial \Omega$,

$$
a_{x x}+\left|a_{y}\right| \leq 0
$$

Then, for a.e. $t \in(0, T)$,

$$
\int_{\Omega}|u(x, y, t)-v(x, y, t)| d x d y \leq c \int_{\Omega}\left|u_{0}(x, y)-v_{0}(x, y)\right| d x d y
$$

We give a simple comment here. In the first place, there do exist domains satisfying the conditions (1.10), (1.11). For example, if the domain $\Omega$ is the unit disc $D_{1}=\{(x, y)$ : 


$$
\begin{aligned}
x^{2}+y^{2} & <1\}, \text { then } a(x, y)=1-\left(x^{2}+y^{2}\right), \\
a_{x} & =-2 x, \quad a_{y}=-2 y, \quad a_{x x}=-2,
\end{aligned}
$$

and

$$
a_{x x}+\left|a_{y}\right| \leq 0 .
$$

In the second place, at least from my own perspective, the conditions (1.10)-(1.11) reflect the regularity of the domain to some extent. For convenience, we can give the following definition.

Definition 1.3 Let $\Omega \subset \mathbb{R}^{2}$ be an open domain. If there is a nonnegative function $g \in$ $C^{1}(\bar{\Omega}), \frac{\partial^{2} g}{\partial x^{2}} \in C(\bar{\Omega})$, such that

$$
\partial \Omega=\left\{(x, y) \in \mathbb{R}^{2}: g(x, y)=0\right\}, \quad \Omega=\left\{(x, y) \in \mathbb{R}^{2}: g(x, y)>0\right\},
$$

and

$$
\frac{\partial^{2} g(x, y)}{\partial x^{2}}+\left|\frac{\partial g(x, y)}{\partial y}\right| \leq 0, \quad(x, y) \in \Omega
$$

then we say $\Omega$ is regular in a special sense.

In other words, Theorem 1.2 reflects that, for a bounded domain $\Omega$, we may use the regularity of the domain in the sense of Definition 1.3 to take the place of the usual boundary value condition.

The second aim of this paper is to study the corresponding problem in an unbounded domain $\Omega$. For any $R \geq 0$, we denote $D_{R}=\left\{(x, y): x^{2}+y^{2}<R^{2}\right\}$ and $\Omega_{R}=\Omega \cap D_{R}$. Inspired by Definition 1.3, we give the following definition.

Definition 1.4 For an unbounded domain $\Omega \subset \mathbb{R}^{2}$, if there exist a constant $R_{0}$ and a nonnegative function $g \in C^{1}(\bar{\Omega})$ satisfying (1.13), $\frac{\partial^{2} g}{\partial x^{2}} \in C(\bar{\Omega})$, such that inequality (1.14) is true when $(x, y) \in \Omega \backslash \Omega_{R_{0}}$, then we say $\Omega$ is weakly regular in a special sense.

It would be perfect if we could prove a theorem similar to Theorem 1.2 when $\Omega$ is unbounded and (weakly) regular. But it seems very difficult. We can only give a partial positive answer. We will provide the definition of the entropy solution to Eq. (1.1) in an unbounded domain in Sect. 3. We establish first its existence and then we can prove the following stability theorems.

Theorem 1.5 Let the unbounded $\Omega$ be weakly regular in the sense of Definition 1.4. Let $0 \leq u \leq 1,0 \leq v \leq 1$ be two solutions of Eq. (1.1) with different initial values $u_{0}, v_{0} \in L^{\infty}(\Omega)$. If $f(\cdot, u)$ is Lipschitz function, and

$$
\begin{aligned}
& g(x, y)\left[\delta^{2} x^{2} \sqrt{1+x^{2}+y^{2}}-\delta\left(1+y^{2}\right)\right] \\
& \quad-2 \delta x\left(1+x^{2}+y^{2}\right) g_{x}+\delta\left|y g_{y}\right|\left(1+x^{2}+y^{2}\right) \leq 0, \quad x \in \Omega \backslash \Omega_{R_{0}},
\end{aligned}
$$




$$
\left|-2 \delta \frac{x g_{x}}{\sqrt{1+x^{2}+y^{2}}}+g_{x x}+\delta \frac{y g_{y}}{\sqrt{1+x^{2}+y^{2}}}+g_{y}\right| \leq c g(x, y), \quad x \in \Omega_{R_{0}}
$$

then, for a.e. $t \in(0, T)$,

$$
\begin{aligned}
& \int_{\Omega}|u(x, y, t)-v(x, y, t)| v_{\delta}(x) g(x, y) d x d y \\
& \quad \leq c \int_{\Omega}\left|u_{0}(x, y)-v_{0}(x, y)\right| v_{\delta}(x) g(x, y) d x d y,
\end{aligned}
$$

where $\delta$ is a given positive constant, and

$$
v_{\delta}(x)=\exp \left(-\delta \sqrt{1+x^{2}+y^{2}}\right)
$$

Moreover, from the proof Theorem 1.5, we are easily to obtain the following.

Theorem 1.6 Let the unbounded $\Omega$ be regular in the sense of Definition 1.3 (equivalently, $R_{0}=0$ in Definition 1.4). Let $0 \leq u \leq 1,0 \leq v \leq 1$ be two solutions of Eq. (1.1) with different initial values $u_{0}, v_{0} \in L^{\infty}(\Omega)$. Iff $(\cdot, u)$ is a Lipschitz function, $g(x, y)$ satisfies

$$
\begin{aligned}
& g(x, y)\left[\delta^{2} x^{2} \sqrt{1+x^{2}+y^{2}}-\delta\left(1+y^{2}\right)\right] \\
& \quad-2 \delta x\left(1+x^{2}+y^{2}\right) g_{x}+\delta\left|y g_{y}\right|\left(1+x^{2}+y^{2}\right) \leq 0, \quad x \in \Omega,
\end{aligned}
$$

then the stability (1.17) is true.

Theorem 1.7 Let $\Omega$ be an unbounded domain in $\mathbb{R}^{2}$. Let $0 \leq u \leq 1,0 \leq v \leq 1$ be two solutions of Eq. (1.1) with the different initial values $u_{0}, v_{0} \in L^{\infty}(\Omega)$. Iff $(\cdot, u)$ is a Lipschitz function, and there exists a nonnegative function $g \in C^{1}(\bar{\Omega}), \frac{\partial^{2} g}{\partial x^{2}} \in C(\bar{\Omega})$, such that (1.13) is true and

$$
\left|-2 \delta \frac{x g_{x}}{\sqrt{1+x^{2}+y^{2}}}+g_{x x}+\delta \frac{y g_{y}}{\sqrt{1+x^{2}+y^{2}}}+g_{y}\right| \leq \operatorname{cg}(x, y), \quad x \in \Omega,
$$

then the stability (1.17) is true.

The limitation of previous theorems is that it is not easy to judge whether the conditions (1.15) (similarly (1.18)) and (1.16) (similarly (1.19)) are true or not. In other words, for a given unbounded regular domain $\Omega$, it is difficult to verify that $\Omega$ is regular (or weakly regular).

Certainly, if one insists on considering the well-posedness problem for Eq. (1.1) in the framework of the initial-boundary value conditions system, then the usual boundary value condition (1.3) may be overdetermined, only a partial boundary value condition is enough. The third aim of this paper is to prove the stability of entropy solutions to Eq. (1.1) based on a partial boundary value condition. For simplicity, we only consider the special unbounded domain

$$
\Omega_{1}=\left\{(x, y) \in \mathbb{R}_{+}^{2}:-x^{2}+y>0\right\}
$$

in this paper. 
Theorem 1.8 Let $0 \leq u \leq 1,0 \leq v \leq 1$ be two solutions of Eq. (1.1) with the different initial values $u_{0}, v_{0} \in L^{\infty}\left(\Omega_{1}\right)$ and with the same partial boundary value condition

$$
u(x, t)=v(x, t)=0, \quad(x, t) \in \Sigma_{1} \times(0, T),
$$

where $\Sigma_{1}=\left\{x \in \partial \Omega_{1}: \frac{2 x_{1}^{2}}{\sqrt{1+x_{1}^{2}+x_{2}^{2}}}-1>0\right\}$. If $f(\cdot, u)$ is a Lipschitz function, then for a.e. $t \in$ $(0, T)$,

$$
\int_{\Omega_{1}}|u(x, y, t)-v(x, y, t)| v_{1}(x) d x d y \leq c \int_{\Omega_{1}}\left|u_{0}(x, y)-v_{0}(x, y)\right| v_{1}(x) d x d y .
$$

We think that Theorem 1.8 can be generalized to a usual unbounded domain, and we will pursue this track further.

\section{The proof of Theorem 1.2}

In this section, we give the proof of Theorem 1.2. Let us recall Kružkov's bi-variable method briefly.

Let $u, v$ be two entropy solutions to Eq. (1.1) with initial values

$$
u(x, y, 0)=u_{0}(x, y), \quad v(x, y, 0)=v_{0}(x, y) .
$$

In order to use Kružkov's bi-variable method, we denote the spatial variables $(x, y)$ by $\left(x_{1}, x_{2}\right)$ or $\left(y_{1}, y_{2}\right)$, and $d x=d x_{1} d x_{2}, d y=d y_{1} d y_{2}$.

By Definition 1.1, we have

$$
\begin{aligned}
& \iint_{Q_{T}}\left[I_{\eta}(u-k) \varphi_{t}-B_{\eta}(u, k) \varphi_{x_{2}}+I_{\eta}(u-k) \varphi_{x_{1} x_{1}}-S_{\eta}^{\prime}(u-k)\left|\partial_{x_{1}} u\right|^{2} \varphi\right] d x d t \\
& \quad-\iint_{Q_{T}} f(\cdot, u) S_{\eta}(u-k) \varphi d x d t \\
& \quad \geq 0 \\
& \iint_{Q_{T}}\left[I_{\eta}(v-l) \varphi_{\tau}-B_{\eta}(v, l) \varphi_{y_{2}}+I_{\eta}(v-l) \varphi_{y_{1} y_{1}}-S_{\eta}^{\prime}(v-l)\left|\partial_{y_{1}} v\right|^{2} \varphi\right] \\
& \quad-\iint_{Q_{T}} f(\cdot, v) S_{\eta}(v-l) \varphi d y d \tau \\
& \quad \geq 0 .
\end{aligned}
$$

Let $\phi \geq 0, \phi \in C_{0}^{\infty}\left(Q_{T}\right), \psi(x, t, y, \tau)=\phi(x, t) j_{h}(x-y, t-\tau)$. Here,

$$
j_{h}(x-y, t-\tau)=\omega_{h}(t-\tau) \prod_{i=1}^{2} \omega_{h}\left(x_{i}-y_{i}\right)
$$

where $\omega_{h}$ is taken as

$$
\omega_{h}(s)=\frac{1}{h} \omega\left(\frac{s}{h}\right), \quad \omega \in C_{0}^{\infty}(\mathbb{R}), \omega \geq 0, \omega=0 \text { if }|s|>1, \int_{-\infty}^{\infty} \omega(s) d s=1 .
$$


Clearly,

$$
\begin{aligned}
& \frac{\partial j_{h}}{\partial t}+\frac{\partial j_{h}}{\partial \tau}=0, \quad \frac{\partial j_{h}}{\partial x_{i}}+\frac{\partial j_{h}}{\partial y_{i}}=0, \quad i=1,2 ; \\
& \frac{\partial \psi}{\partial t}+\frac{\partial \psi}{\partial \tau}=\frac{\partial \phi}{\partial t} j_{h}, \quad \frac{\partial \psi}{\partial x_{i}}+\frac{\partial \psi}{\partial y_{i}}=\frac{\partial \phi}{\partial x_{i}} j_{h} .
\end{aligned}
$$

By choosing $k=v(y, \tau)$ in (2.2), $l=u(x, t)$ in (2.3), and $\varphi=\psi(x, t, y, \tau)$ in both (2.2) and (2.3), we have

$$
\begin{aligned}
& \iint_{Q_{T}} \iint_{Q_{T}}\left[I_{\eta}(u-v)\left(\psi_{t}+\psi_{\tau}\right)-\left(B_{\eta}(u, v) \psi_{x_{2}}+B_{\eta}(v, u) \psi_{y_{2}}\right)\right. \\
& \left.\quad+I_{\eta}(u-v) \psi_{x_{1} x_{1}}+I_{\eta}(v-u) \psi_{y_{1} y_{1}}\right] \\
& \quad-\left\{S_{\eta}^{\prime}(u-v)\left(\left|\partial_{x_{1}} u\right|^{2}+\left|\partial_{y_{1}} v\right|^{2}\right)\right. \\
& \left.\quad-\left[f(\cdot, u) S_{\eta}(u-v)+f(\cdot, v) S_{\eta}(v-u)\right]\right\} \varphi d x d t d y d \tau \\
& \quad \geq 0 .
\end{aligned}
$$

By (2.4)-(2.5), using the equality (2.5), we can deduce that

$$
\left(u^{+}-u^{-}\right) v_{1}=0, \quad(x, t) \in \Gamma_{u},
$$

where $\bar{v}=\left(v_{1}, v_{2}, v_{3}\right)$ is the normal vector of $\Gamma_{u}$ at the point $(x, y, t)$. By (2.7), after a calculation developed in [9], when $\eta \rightarrow 0$ and then $h \rightarrow 0^{+}$in (2.6), we get

$$
\begin{aligned}
& \iint_{Q_{T}}\left[|u(x, t)-v(x, t)| \phi_{t}+|u-v| \phi_{x_{1} x_{1}}-\frac{1}{2} \operatorname{sgn}(u-v)\left(u^{2}-v^{2}\right) \phi_{x_{2}}\right] d x d t \\
& \quad-\iint_{Q_{T}}[f(\cdot, u)-f(\cdot, v)] \operatorname{sgn}(u-v) \phi d x d t \\
& \quad \geq 0 .
\end{aligned}
$$

If we choose

$$
\phi(x, t)=\eta(t) \xi(x)
$$

where $0 \leq \eta \in C_{0}^{\infty}(0, T), 0 \leq \xi \in C_{0}^{\infty}(\Omega)$, then

$$
\begin{aligned}
\iint_{Q_{T}} & {\left[|u(x, t)-v(x, t)| \xi(x) \eta_{t}+|u-v| \eta(t) \xi_{x_{1} x_{1}}\right.} \\
& \left.-\frac{1}{2} \operatorname{sgn}(u-v)\left(u^{2}-v^{2}\right) \eta(t) \xi_{x_{2}}\right] d x d t \\
& -\iint_{Q_{T}}[f(\cdot, u)-f(\cdot, v)] \operatorname{sgn}(u-v) \eta(t) \xi(x) d x d t
\end{aligned}
$$

$\geq 0$. 
For $0<\tau<s<T$, we choose

$$
\eta(t)=\int_{\tau-t}^{s-t} \omega_{\epsilon}(\sigma) d \sigma, \quad \epsilon<\min \{\tau, T-s\}
$$

where, as above, $\omega_{\epsilon}(t)$ is the kernel of the mollifier with $\omega_{\epsilon}(t)=0$ for $t \notin(-\epsilon, \epsilon)$.

By (2.9), letting $\varepsilon \rightarrow 0$, we have

$$
\begin{aligned}
& \int_{\Omega}|u(x, s)-v(x, s)| \xi(x) d x \\
& \leq \int_{\Omega}|u(x, \tau)-v(x, \tau)| \xi(x) d x \\
& \quad+\int_{\tau}^{s} \int_{\Omega}\left\{|u-v| \xi_{x_{1} x_{1}}-\frac{u+v}{2}|u-v| \xi_{x_{2}}\right. \\
& \quad-[f(\cdot, u)-f(\cdot, v)] \operatorname{sgn}(u-v) \xi(x)\} d x d t .
\end{aligned}
$$

For any given small enough $0<\lambda, \omega_{\lambda} \in C_{0}^{1}(\Omega)$ is defined as follows:

$$
\omega_{\lambda}(x)= \begin{cases}1, & a(x)>\lambda, \\ 1-\frac{(a(x)-\lambda)^{2}}{\lambda^{2}}, & 0 \leq a(x) \leq \lambda,\end{cases}
$$

where $a$ satisfies the conditions (1.10)-(1.11) and $a_{x_{1} x_{1}} \in C(\bar{\Omega})$.

We choose now $\xi(x)=\omega_{\lambda}(x)$. So, for $0 \leq a(x) \leq \lambda$,

$$
\begin{aligned}
& \xi_{x_{1}}=-\frac{2(a-\lambda)}{\lambda^{2}} a_{x_{1}}, \quad \xi_{x_{2}}=-\frac{2(a-\lambda)}{\lambda^{2}} a_{x_{2}}, \\
& \xi_{x_{1} x_{1}}=-\frac{2}{\lambda^{2}} a_{x_{1}}^{2}-\frac{2(a-\lambda)}{\lambda^{2}} a_{x_{1} x_{1}} .
\end{aligned}
$$

Then

$$
\begin{aligned}
& \int_{\Omega}|u(x, s)-v(x, s)| \xi(x) d x \\
& \leq \quad \int_{\Omega}|u(x, \tau)-v(x, \tau)| \xi(x) d x \\
& \quad+\int_{\tau}^{s} \int_{\Omega_{\lambda}}\left\{-|u-v| \frac{2(a-\lambda)}{\lambda^{2}} a_{x_{1} x_{1}}+\frac{u+v}{2}|u-v| \frac{2(a-\lambda)}{\lambda^{2}} a_{x_{2}}\right\} d x d t \\
& \quad-\int_{\tau}^{s} \int_{\Omega}[f(\cdot, u)-f(\cdot, v)] \operatorname{sgn}(u-v) \omega_{\lambda}(x) d x d t
\end{aligned}
$$

where $\Omega_{\lambda}=\{x \in \Omega: a(x)<\lambda\}$.

Since $a$ satisfies the conditions (1.10)-(1.11), using the fact $0 \leq u \leq 1,0 \leq v \leq 1$, we have

$$
\begin{aligned}
& \frac{u+v}{2}|u-v| \frac{2(a-\lambda)}{\lambda^{2}} a_{x_{2}} \\
& \quad \leq \frac{u+v}{2}|u-v|\left|\frac{2(a-\lambda)}{\lambda^{2}}\right|\left|a_{x_{2}}\right|
\end{aligned}
$$




$$
\begin{aligned}
& \leq|u-v|\left|\frac{2(a-\lambda)}{\lambda^{2}}\right|\left|a_{x_{2}}\right| \\
& =-|u-v| \frac{2(a-\lambda)}{\lambda^{2}}\left|a_{x_{2}}\right|, \quad x \in \Omega_{\lambda},
\end{aligned}
$$

accordingly,

$$
\begin{aligned}
& -|u-v| \frac{2(a-\lambda)}{\lambda^{2}} a_{x_{1} x_{1}}+\frac{u+v}{2}|u-v| \frac{2(a-\lambda)}{\lambda^{2}} a_{x_{2}} \\
& \leq-|u-v| \frac{2(a-\lambda)}{\lambda^{2}}\left(a_{x_{1} x_{1}}+\left|a_{x_{2}}\right|\right) \\
& \leq 0, x \in \Omega_{\lambda},
\end{aligned}
$$

and by (2.11), we have

$$
\int_{\Omega}|u(x, s)-v(x, s)| d x \leq \int_{\Omega}|u(x, \tau)-v(x, \tau)| d x+c \int_{\tau}^{s} \int_{\Omega}|u-v| d x d t .
$$

By the Gronwall lemma,

$$
\int_{\Omega}|u(x, s)-v(x, s)| d x \leq c^{*} \int_{\Omega}|u(x, \tau)-v(x, \tau)| d x
$$

where $c^{*}=e^{c T}$. After letting $\tau \rightarrow 0$ in (2.13), we have

$$
\int_{\Omega}|u(x, s)-v(x, s)| d x \leq c^{*} \int_{\Omega}\left|u_{0}(x)-v_{0}(x)\right| d x
$$

\section{Unbounded domains}

If $\Omega$ is an unbounded domain, we should modify the definition of entropy solutions as follows.

Definition 3.1 A function $u$ is said to be the entropy solution of Eq. (1.1) with the initial value (1.2), if $u$ satisfies (i) and (ii) in Definition 1.1, and the initial value is true in the sense that

$$
\lim _{t \rightarrow 0} \int_{\Omega_{R}}\left|u(x, t)-u_{0}(x)\right| d x=0
$$

where $\Omega_{R}=D_{R} \cap \Omega$.

Since the domain $\Omega$ is unbounded, the existence of the entropy solution in the sense of Definition 3.1, should be proved independently. In what follows, we only give the outline of its proof.

Consider the approximate problem,

$$
\begin{aligned}
& \varepsilon \Delta u_{\varepsilon}+\partial_{x x} u_{\varepsilon}+u_{\varepsilon} \partial_{y} u_{\varepsilon}-\partial_{t} u_{\varepsilon}=f\left(x, y, t, u_{\varepsilon}\right), \quad(x, y, t) \in \Omega_{\frac{1}{\varepsilon}} \times(0, T) \\
& u_{\varepsilon}(x, t)=0, \quad(x, t) \in \partial \Omega_{\frac{1}{\varepsilon}} \times(0, T) \\
& u_{\varepsilon}(x, 0)=u_{0}(x), \quad x \in \Omega_{\frac{1}{\varepsilon}}
\end{aligned}
$$


where $\Omega_{\frac{1}{\varepsilon}}=D_{\frac{1}{\varepsilon}} \cap \Omega$. We assume $0 \leq u_{0} \in C_{0}^{2}(\Omega)$. It is well known that there is a classical solution $u_{\varepsilon}$.

Denote $x_{1}=x, x_{2}=y, x_{3}=t$, differentiate (3.5) with respect to $x_{s}, s=1,2,3$, and sum up for $s$ after multiplying the resulting relation by $u_{x_{s}} \frac{S_{\eta}\left(\left|\operatorname{grad} u_{\varepsilon}\right|\right)}{\left|\operatorname{grad} u_{\varepsilon}\right|} v_{\delta}(x)$, by a complicated calculation, similar to those in [9], then we can deduce the following important lemma.

Lemma 3.2 Iff is a $C^{1}$ function, $f_{x_{1}}, f_{x_{2}}, f_{t}$ are bounded functions, and $f_{u}$ is bounded too when $u$ is bounded, then we have

$$
\begin{aligned}
& \int_{0}^{T} \int_{\Omega}\left|\operatorname{grad} u_{\varepsilon}\right| v_{\delta}(x) d x d t \leq c, \\
& \int_{0}^{T} \int_{\Omega_{1}} v_{\delta}(x)\left|u_{x_{1}}\right|^{2} d x d t \leq c .
\end{aligned}
$$

Theorem 3.3 If $0 \leq u_{0} \in C_{0}^{2}(\Omega)$, $f$ is a $C^{1}$ function, $f_{x_{1}}, f_{x_{2}}, f_{t}$ are bounded functions, and $f_{u}$ is bounded when $u$ is bounded, there exists an entropy solution of Eq. (1.1) in the sense of Definition 3.1.

Proof By Kolmogoroff's theorem, there exist a subsequence $\left\{u_{\varepsilon_{n}}\right\}$ of $u_{\varepsilon}$ and a function $u \in \operatorname{BV}\left(Q_{T}\right) \cap L^{\infty}\left(Q_{T}\right)$ such that $\left(u_{\varepsilon_{\varepsilon}}\right)$ is locally strongly convergent in $L^{1}$ to $\mathrm{u}$, and so $u_{\varepsilon_{\varepsilon}} \rightarrow u$ a.e. on $Q_{T}$. By (3.6), there exists a subsequence of $\{\varepsilon\}$ (we still denote it $\varepsilon$ ), such that

$$
\frac{\partial u_{\varepsilon}}{\partial x} \rightarrow \frac{\partial u}{\partial x}, \quad \text { in } L_{\mathrm{loc}}^{2}\left(Q_{T}\right)
$$

Let $\varphi \in C_{0}^{2}\left(Q_{T}\right), \varphi \geq 0$. Multiplying (3.4) by $\varphi S_{\eta}\left(u_{\varepsilon}-k\right)$, and integrating over $Q_{T}$, we obtain

$$
\begin{aligned}
& \iint_{Q_{T}} \frac{\partial u_{\varepsilon}}{\partial t} \varphi S_{\eta}\left(u_{\varepsilon}-k\right) d x d y d t \\
& =\iint_{Q_{T}} \frac{\partial}{\partial x}\left(\frac{\partial u_{\varepsilon}}{\partial x}\right) \varphi S_{\eta}\left(u_{\varepsilon}-k\right) d x d y d t \\
& \quad+\varepsilon \iint_{Q_{T}} \Delta u_{\varepsilon} \varphi S_{\eta}\left(u_{\varepsilon}-k\right) d x d y d t+\iint_{Q_{T}} u_{\varepsilon} u_{\varepsilon y} \varphi S_{\eta}\left(u_{\varepsilon}-k\right) d x d y d t \\
& \quad-\iint_{Q_{T}} f\left(x, y, t, u_{\varepsilon}\right) \varphi S_{\eta}\left(u_{\varepsilon}-k\right) d x d y d t
\end{aligned}
$$

Integrating by parts, we can deduce that

$$
\begin{aligned}
& \iint_{Q_{T}} I_{\eta}\left(u_{\varepsilon}-k\right) \varphi_{t} d x d y d t+\iint_{Q_{T}} I_{\eta}\left(u_{\varepsilon}-k\right) \varphi_{x x} d x d y d t-\iint_{Q_{T}} B_{\eta}\left(u_{\varepsilon}, k\right) \varphi_{y} d x d y d t \\
& \quad-\varepsilon \iint_{Q_{T}} \nabla u_{\varepsilon} \cdot \nabla \varphi S_{\eta}\left(u_{\varepsilon}-k\right) d x d y d t-\varepsilon \iint_{Q_{T}}\left|\nabla u_{\varepsilon}\right|^{2} S_{\eta}^{\prime}\left(u_{\varepsilon}-k\right) \varphi d x d y d t \\
& \quad-\iint_{Q_{T}}\left(u_{\varepsilon x}\right)^{2} S_{\eta}^{\prime}\left(u_{\varepsilon}-k\right) \varphi d x d y d t-\iint_{Q_{T}} f\left(x, y, t, u_{\varepsilon}\right) \varphi S_{\eta}\left(u_{\varepsilon}-k\right) d x d y d t \\
& \quad=0
\end{aligned}
$$


Accordingly, we have

$$
\begin{aligned}
& \iint_{Q_{T}} I_{\eta}\left(u_{\varepsilon}-k\right) \varphi_{t} d x d y d t+\iint_{Q_{T}} I_{\eta}\left(u_{\varepsilon}-k\right) \varphi_{x x} d x d y d t-\iint_{Q_{T}} B_{\eta}\left(u_{\varepsilon}, k\right) \varphi_{y} d x d y d t \\
& \quad-\varepsilon \iint_{Q_{T}} \nabla u_{\varepsilon} \cdot \nabla \varphi S_{\eta}\left(u_{\varepsilon}-k\right) d x d y d t-\iint_{Q_{T}}\left(u_{\varepsilon x}\right)^{2} S_{\eta}^{\prime}\left(u_{\varepsilon}-k\right) \varphi d x d y d t \\
& \quad-\iint_{Q_{T}} f\left(x, y, t, u_{\varepsilon}\right) \varphi S_{\eta}\left(u_{\varepsilon}-k\right) d x d t \\
& \quad \geq 0
\end{aligned}
$$

We use the inequality

$$
\liminf _{\varepsilon \rightarrow 0} \iint_{Q_{T}} S_{\eta}^{\prime}\left(u_{\varepsilon}-k\right) \frac{\partial u_{\varepsilon}}{\partial x} \frac{\partial u_{\varepsilon}}{\partial x} \varphi d x d y d t \geq \iint_{Q_{T}}\left|\frac{\partial u}{\partial x}\right|^{2} S_{\eta}^{\prime}(u-k) \varphi d x d y d t .
$$

Let $\varepsilon \rightarrow 0$ in (3.10). By (3.11), we know $u_{\varepsilon} \rightarrow u$ and $u$ satisfies (i) and (ii) in Definition 1.1.

The initial value condition, satisfied in the sense of (3.3), can be proved by a similar method to that in [7]. Then $u$ is an entropy solution of Eq. (1.1) in the sense of Definition 3.1.

For convenience, we denote again $(x, y)=\left(x_{1}, x_{2}\right)$. Theorem 1.5 is proved as follows.

Proof of Theorem 1.5 Let us choose the test function

$$
\phi(t, x)=\eta(t) \xi(x)
$$

and using Kružkov's bi-variable method we obtain

$$
\begin{aligned}
& \int_{\Omega}|u(x, s)-v(x, s)| \xi(x) d x \\
& \leq \int_{\Omega}|u(x, \tau)-v(x, \tau)| \xi(x) d x \\
& \quad+\int_{s}^{\tau} \int_{\Omega}\left\{|u-v| \xi_{x_{1} x_{1}}-\frac{u+v}{2}|u-v| \xi_{x_{2}}\right. \\
& \quad-[f(\cdot, u)-f(\cdot, v)] \operatorname{sgn}(u-v) \xi(x)\} d x d t .
\end{aligned}
$$

By taking the limit, we can choose

$$
\xi\left(x_{1}, x_{2}\right)=v_{\delta}\left(x_{1}, x_{2}\right) g\left(x_{1}, x_{2}\right),
$$

where $g\left(x_{1}, x_{2}\right)$ satisfies the conditions (1.14)-(1.16).

In the first place, we have the following direct calculations:

$$
\begin{aligned}
& v_{\delta}=e^{-\delta \sqrt{1+x_{1}^{2}+x_{2}^{2}}}, \\
& v_{\delta x_{1}}=-\delta v_{\delta} \frac{x_{1}}{\sqrt{1+x_{1}^{2}+x_{2}^{2}}},
\end{aligned}
$$




$$
\begin{aligned}
v_{\delta x_{2}}= & -\delta v_{\delta} \frac{x_{2}}{\sqrt{1+x_{1}^{2}+x_{2}^{2}}}, \\
v_{\delta x_{1} x_{1}}= & \delta^{2} v_{\delta} \frac{x_{1}^{2}}{1+x_{1}^{2}+x_{2}^{2}}-\delta v_{\delta} \frac{1+x_{2}^{2}}{\left(1+x_{1}^{2}+x_{2}^{2}\right)^{\frac{3}{2}}}, \\
\xi_{x_{2}}= & v_{\delta x_{2}} g\left(x_{1}, x_{2}\right)+v_{\delta} g_{x_{2}}\left(x_{1}, x_{2}\right) \\
= & -\delta v_{\delta} \frac{x_{2} g\left(x_{1}, x_{2}\right)}{\sqrt{1+x_{1}^{2}+x_{2}^{2}}}+v_{\delta} g_{x_{2}}, \\
\xi_{x_{1} x_{1}}= & v_{\delta x_{1} x_{1} g\left(x_{1}, x_{2}\right)+2 v_{\delta x_{1}} g_{x_{1}}+v_{\delta} g_{x_{1} x_{1}}} \\
= & {\left[\delta v_{\delta}^{2} \frac{x_{1}^{2}}{1+x_{1}^{2}+x_{2}^{2}}-\delta v_{\delta} \frac{1+x_{2}^{2}}{\left(1+x_{1}^{2}+x_{2}^{2}\right)^{\frac{3}{2}}}\right] g\left(x_{1}, x_{2}\right) } \\
& -2 \delta v_{\delta} \frac{x_{1} g_{x_{1}}}{\sqrt{1+x_{1}^{2}+x_{2}^{2}}}+v_{\delta} g_{x_{1} x_{1}}\left(x_{1}, x_{2}\right) .
\end{aligned}
$$

Since the domain $\Omega$ is weakly regular in the sense of Definition 1.4 , there is a $R_{0} \geq 0$,

$$
\Omega_{R_{0}}=\left\{x \in \Omega,|x|^{2}<R_{0}^{2}\right\}
$$

such that when $x \in \Omega \backslash \Omega_{R_{0}}$, (1.14) is true, i.e.

$$
g_{x_{1} x_{1}}+\left|g_{x_{2}}\right| \leq 0
$$

In the second place, we use these calculations (3.19)-(3.21) in (3.13).

$$
\begin{aligned}
& \int_{\Omega}\left(|u-v| \xi_{x_{1} x_{1}}-\frac{u+v}{2}|u-v| \xi_{x_{2}}\right) d x \\
& \quad=\left(\int_{\Omega_{R_{0}}}+\int_{\Omega \backslash \Omega_{R_{0}}}\right)|u-v|\left(\xi_{x_{1} x_{1}}-\frac{u+v}{2} \xi_{x_{2}}\right) d x .
\end{aligned}
$$

Since $0 \leq u \leq 1,0 \leq v \leq 1$, we have

$$
\begin{aligned}
& \int_{\Omega \backslash \Omega_{R_{0}}}|u-v|\left(\xi_{x_{1} x_{1}}-\frac{u+v}{2} \xi_{x_{2}}\right) d x \\
& =\int_{\Omega \backslash \Omega_{R_{0}}}|u-v|\left\{\left[\delta^{2} v_{\delta} \frac{x_{1}^{2}}{1+x_{1}^{2}+x_{2}^{2}}-\delta v_{\delta} \frac{1+x_{2}^{2}}{\left(1+x_{1}^{2}+x_{2}^{2}\right)^{\frac{3}{2}}}\right] g\left(x_{1}, x_{2}\right)\right. \\
& \quad-2 \delta v_{\delta} \frac{x_{1} g_{x_{1}}}{\sqrt{1+x_{1}^{2}+x_{2}^{2}}}+v_{\delta} g_{x_{1} x_{1}} \\
& \left.\quad+\frac{u+v}{2} \delta v_{\delta} \frac{x_{2} g_{x_{2}}}{\sqrt{1+x_{1}^{2}+x_{2}^{2}}}+v_{\delta} g_{x_{2}}\right\} d x \\
& \leq \int_{\Omega \backslash \Omega_{R_{0}}} \frac{v_{\delta}|u-v|}{\left(1+x_{1}^{2}+x_{2}^{2}\right)^{\frac{3}{2}}}\left\{g\left(x_{1}, x_{2}\right)\left[\delta^{2} x_{1}^{2} \sqrt{1+x_{1}^{2}+x_{2}^{2}}-\delta\left(1+x_{2}^{2}\right)\right]\right.
\end{aligned}
$$




$$
\begin{aligned}
& \left.\quad-2 \delta x_{1}\left(1+x_{1}^{2}+x_{2}^{2}\right) g_{x_{1}}+\delta\left|x_{2} g_{x_{2}}\right|\left(1+x_{1}^{2}+x_{2}^{2}\right)\right\} d x \\
& \quad+\int_{\Omega \backslash \Omega_{R_{0}}} v_{\delta}\left(g_{x_{1} x_{1}}+\left|g_{x_{2}}\right|\right) d x \\
& \leq
\end{aligned}
$$

Here, we have use the assumption (1.15)

$$
\begin{aligned}
& g\left(x_{1}, x_{2}\right)\left[\delta^{2} x_{1}^{2} \sqrt{1+x_{1}^{2}+x_{2}^{2}}-\delta\left(1+x_{2}^{2}\right)\right] \\
& -2 \delta x_{1}\left(1+x_{1}^{2}+x_{2}^{2}\right) g_{x_{1}}+\delta\left|x_{2} g_{x_{2}}\right|\left(1+x_{1}^{2}+x_{2}^{2}\right) \leq 0, \quad x \in \Omega \backslash \Omega_{R_{0}},
\end{aligned}
$$

and since the domain $\Omega$ is weakly regular,

$$
g_{x_{1} x_{1}}+\left|g_{x_{2}}\right| \leq 0, \quad x \in \Omega \backslash \Omega_{R_{0}} .
$$

Meanwhile,

$$
\begin{aligned}
& \int_{\Omega_{R_{0}}}|u-v|\left(\xi_{x_{1} x_{1}}-\frac{u+v}{2} \xi_{x_{2}}\right) d x \\
& =\int_{\Omega_{R_{0}}}|u-v|\left\{\left[\delta^{2} v_{\delta} \frac{x_{1}^{2}}{1+x_{1}^{2}+x_{2}^{2}}-\delta v_{\delta} \frac{1+x_{2}^{2}}{\left(1+x_{1}^{2}+x_{2}^{2}\right)^{\frac{3}{2}}}\right] g\left(x_{1}, x_{2}\right)\right. \\
& \quad-2 \delta v_{\delta} \frac{x_{1} g_{x_{1}}}{\sqrt{1+x_{1}^{2}+x_{2}^{2}}}+v_{\delta} g_{x_{1} x_{1}} \\
& \left.\quad+\frac{u+v}{2} \delta v_{\delta} \frac{x_{2} g_{x_{2}}}{\sqrt{1+x_{1}^{2}+x_{2}^{2}}}+v_{\delta} g_{x_{2}}\right\} d x \\
& \leq \int_{\Omega_{R_{0}}}|u-v|\left\{\left[\delta^{2} \frac{x_{1}^{2}}{1+x_{1}^{2}+x_{2}^{2}}-\delta \frac{1+x_{2}^{2}}{\left(1+x_{1}^{2}+x_{2}^{2}\right)^{\frac{3}{2}}}\right] g\left(x_{1}, x_{2}\right) v_{\delta}\right. \\
& \left.\quad+v_{\delta}\left|-2 \delta \frac{x_{1} g_{x_{1}}}{\sqrt{1+x_{1}^{2}+x_{2}^{2}}}+g_{x_{1} x_{1}}+\delta \frac{x_{2} g_{x_{2}}}{\sqrt{1+x_{1}^{2}+x_{2}^{2}}}+g_{x_{2}}\right|\right\} d x \\
& \leq c \int_{\Omega_{R_{0}}}|u-v| g\left(x_{1}, x_{2}\right) v_{\delta} d x .
\end{aligned}
$$

Here, we have used the assumption (1.16)

$$
\left|-2 \delta \frac{x_{1} g_{x_{1}}}{\sqrt{1+x_{1}^{2}+x_{2}^{2}}}+g_{x_{1} x_{1}}+\delta \frac{x_{2} g_{x_{2}}}{\sqrt{1+x_{1}^{2}+x_{2}^{2}}}+g_{x_{2}}\right| \leq c g\left(x_{1}, x_{2}\right), \quad x \in \Omega_{R_{0}} .
$$

At last, we substitute (3.23)-(3.24) into (3.13) to get

$$
\begin{aligned}
& \int_{\Omega}|u(x, s)-v(x, s)| v_{\delta}(x) g\left(x_{1}, x_{2}\right) d x \\
& \quad \leq \int_{\Omega}|u(x, \tau)-v(x, \tau)| v_{\delta}(x) g\left(x_{1}, x_{2}\right) d x+c \int_{\tau}^{s} \int_{\Omega}|u-v| v_{\delta}(x) g\left(x_{1}, x_{2}\right) d x d t .
\end{aligned}
$$


By the Gronwall lemma, we have

$$
\int_{\Omega}|u(x, s)-v(x, s)| v_{\delta}(x) g\left(x_{1}, x_{2}\right) d x \leq c^{*} \int_{\Omega}|u(x, \tau)-v(x, \tau)| v_{\delta}(x) g\left(x_{1}, x_{2}\right) d x,
$$

where $c^{*}=e^{c T}$, and the conclusion follows easily.

From the proof of Theorem 3.3 (Theorem 1.5), we easily obtain Theorem 1.6 and Theorem 1.7 , we omit the details here.

\section{The proof of Theorem 1.8}

In the last section, we prove Theorem 1.8. As before, we choose the test function

$$
\phi(t, x)=\eta(t) \xi(x)
$$

and use Kružkov's bi-variable method to obtain

$$
\begin{aligned}
& \int_{\Omega}|u(x, s)-v(x, s)| \xi(x) d x \\
& \leq \int_{\Omega}|u(x, \tau)-v(x, \tau)| \xi(x) d x \\
& \quad+\int_{\tau}^{s} \int_{\Omega}\left\{|u-v| \xi_{x_{1} x_{1}}-\frac{u+v}{2}|u-v| \xi_{x_{2}}\right. \\
& \quad-[f(\cdot, u)-f(\cdot, v)] \operatorname{sgn}(u-v) \xi(x)\} d x d t .
\end{aligned}
$$

By a process of limit, we can choose $\delta=1$ in the function $v_{\delta}(x)$ and

$$
\xi\left(x_{1}, x_{2}\right)=v_{1}(x) \phi_{\lambda}\left(x_{2}-x_{1}^{2}\right)
$$

where $\lambda>0$ is small enough,

$$
\phi_{\lambda}(t)= \begin{cases}1, & \text { if } t \geq \lambda, \\ 1-\frac{(t-\lambda)^{2}}{\lambda^{2}}, & \text { if } 0<t<\lambda,\end{cases}
$$

Since

$$
\begin{aligned}
& v_{1 x_{1}}=-\frac{x_{1}}{\sqrt{1+x_{1}^{2}+x_{2}^{2}}} v_{1}, \quad \nu_{1 x_{2}}=-\frac{x_{2}}{\sqrt{1+x_{1}^{2}+x_{2}^{2}}} v_{1}, \\
& v_{1 x_{1} x_{1}}=-\frac{1+x_{2}^{2}}{\left(1+x_{1}^{2}+x_{2}^{2}\right)^{\frac{3}{2}}} v_{1}+\frac{x_{1}^{2}}{1+x_{1}^{2}+x_{2}^{2}} v_{1} .
\end{aligned}
$$

Then

$$
\begin{aligned}
\xi_{x_{1}} & =v_{1 x_{1}} \phi_{\lambda}-2 x_{1} \phi_{\lambda}^{\prime} v_{1} \\
& =v_{1}\left[\frac{-x_{1} \phi_{\lambda}}{\sqrt{1+x_{1}^{2}+x_{2}^{2}}}-2 x_{1} \phi_{\lambda}^{\prime}\right],
\end{aligned}
$$


where

$$
\phi_{\lambda}^{\prime}(t)= \begin{cases}0, & \text { if } t \geq \lambda, \\ -\frac{2(t-\lambda))}{\lambda^{2}}, & \text { if } t<\lambda,\end{cases}
$$

and

$$
\begin{aligned}
& \phi_{\lambda}^{\prime \prime}(t)= \begin{cases}0, & \text { if } t \geq \lambda, \\
-\frac{2}{\lambda^{2}}, & \text { if } t<\lambda,\end{cases} \\
& \xi_{x_{2}}=v_{1 x_{2}} \phi_{\lambda}+v_{1} \phi_{\lambda}^{\prime} \\
& =v_{1}\left[\frac{-x_{2} \phi_{\lambda}}{\sqrt{1+x_{1}^{2}+x_{2}^{2}}}+\phi_{\lambda}^{\prime}\right] \text {, }
\end{aligned}
$$

we have

$$
\begin{aligned}
\xi_{x_{1} x_{1}}= & v_{1 x_{1} x_{1}} \phi_{\lambda}-4 x_{1} v_{1 x_{1}} \phi_{\lambda}^{\prime}-2 \phi_{\lambda}^{\prime} \nu_{1}+4 x_{1}^{2} \phi_{\lambda}^{\prime \prime} v_{1} \\
= & {\left[-\frac{1+x_{2}^{2}}{\left(1+x_{1}^{2}+x_{2}^{2}\right)^{\frac{3}{2}}} v_{1}+\frac{x_{1}^{2}}{1+x_{1}^{2}+x_{2}^{2}} v_{1}\right] \phi_{\lambda} } \\
& +4 \frac{x_{1}^{2}}{\sqrt{1+x_{1}^{2}+x_{2}^{2}}} \phi_{\lambda}^{\prime} v_{1} \\
& -2 \phi_{\lambda}^{\prime} v_{1}+4 x_{1}^{2} \phi_{\lambda}^{\prime \prime} v_{1} .
\end{aligned}
$$

Accordingly, by $\phi_{\lambda}^{\prime}(s) \geq 0$ and $\phi_{\lambda}^{\prime \prime}(s) \leq 0$ when $s<\lambda$ is small enough, we extrapolate:

$$
\begin{aligned}
\int_{\Omega} & {\left[|u-v| \xi_{x_{1} x_{1}}-\frac{u+v}{2}|u-v| \xi_{x_{2}}\right] d x } \\
= & \int_{\Omega}|u-v|\left[-\frac{1+x_{2}^{2}}{\left(1+x_{1}^{2}+x_{2}^{2}\right)^{\frac{3}{2}}} v_{1}+\frac{x_{1}^{2}}{1+x_{1}^{2}+x_{2}^{2}} v_{1}\right] \phi_{\lambda} d x \\
& +4 \int_{\Omega}|u-v| \frac{x_{1}^{2}}{\sqrt{1+x_{1}^{2}+x_{2}^{2}}} \phi_{\lambda}^{\prime} v_{1} d x \\
& -2 \int_{\Omega}|u-v|\left[\phi_{\lambda}^{\prime} v_{1}-2 x_{1}^{2} \phi_{\lambda}^{\prime \prime} v_{1}\right] d x \\
& -\int_{\Omega} \frac{u+v}{2}|u-v| v_{1}\left[\frac{-x_{2} \phi_{\lambda}}{\sqrt{1+x_{1}^{2}+x_{2}^{2}}}+\phi_{\lambda}^{\prime}\right] d x \\
= & \int_{\Omega}|u-v|\left[-\frac{1+x_{2}^{2}}{\left(1+x_{1}^{2}+x_{2}^{2}\right)^{\frac{3}{2}}} v_{1}+\frac{x_{1}^{2}}{1+x_{1}^{2}+x_{2}^{2}} v_{1}\right] \phi_{\lambda} d x \\
& +4 \int_{\Omega_{\lambda}}|u-v| \frac{x_{1}^{2}}{\sqrt{1+x_{1}^{2}+x_{2}^{2}}} \phi_{\lambda}^{\prime} v_{1} d x \\
& -2 \int_{\Omega_{\lambda}}|u-v|\left[\phi_{\lambda}^{\prime} v_{1}-2 x_{1}^{2} \phi_{\lambda}^{\prime \prime} v_{1}\right] d x \\
& +\int_{\Omega} \frac{u+v}{2}|u-v| v_{1} \frac{x_{2} \phi_{\lambda}}{\sqrt{1+x_{1}^{2}+x_{2}^{2}}} d x-\int_{\Omega_{\lambda}} \frac{u+v}{2}|u-v| v_{1} \phi_{\lambda}^{\prime} d x
\end{aligned}
$$




$$
\begin{aligned}
\leq & \int_{\Omega}|u-v|\left[-\frac{1+x_{2}^{2}}{\left(1+x_{1}^{2}+x_{2}^{2}\right)^{\frac{3}{2}}} v_{1}+\frac{x_{1}^{2}}{1+x_{1}^{2}+x_{2}^{2}} v_{1}\right] \phi_{\lambda} d x \\
& +4 \int_{\Omega_{\lambda}}|u-v|\left[\frac{4 x_{1}^{2}}{\sqrt{1+x_{1}^{2}+x_{2}^{2}}}-2\right] \phi_{\lambda}^{\prime} v_{1} d x \\
& +\int_{\Omega} \frac{u+v}{2}|u-v| v_{1} \frac{x_{2} \phi_{\lambda}}{\sqrt{1+x_{1}^{2}+x_{2}^{2}}} d x \\
\leq & \int_{\Omega}|u-v|\left[-\frac{1+x_{2}^{2}}{\left(1+x_{1}^{2}+x_{2}^{2}\right)^{\frac{3}{2}}} v_{1}+\frac{x_{1}^{2}}{1+x_{1}^{2}+x_{2}^{2}} v_{1}\right] \phi_{\lambda} d x \\
& +4 \int_{\Omega_{\lambda 1}}|u-v|\left[\frac{4 x_{1}^{2}}{\sqrt{1+x_{1}^{2}+x_{2}^{2}}}-2\right] \phi_{\lambda}^{\prime} v_{1} d x \\
& +\int_{\Omega} \frac{u+v}{2}|u-v| v_{1} \frac{x_{2} \phi_{\lambda}}{\sqrt{1+x_{1}^{2}+x_{2}^{2}}} d x
\end{aligned}
$$

where $\Omega_{\lambda}=\left\{x=\left(x_{1}, x_{2}\right) \in \Omega: x_{2}-x_{1}^{2}<\lambda\right\}, \Omega_{\lambda 1}=\left\{x \in \Omega_{\lambda}: \frac{2 x_{1}^{2}}{\sqrt{1+x_{1}^{2}+x_{2}^{2}}}-1>0\right\}$.

Since $u=v=0$ when $x \in \Sigma_{1}$, using the fact

$$
0<-\left(x_{2}-x_{1}^{2}-\lambda\right)=\lambda-\left(x_{2}-x_{1}^{2}\right)<\lambda
$$

we have

$$
\begin{aligned}
& \lim _{\lambda \rightarrow 0} \int_{\Omega_{\lambda 1}}|u-v|\left[\frac{4 x_{1}^{2}}{\sqrt{1+x_{1}^{2}+x_{2}^{2}}}-2\right] \phi_{\lambda}^{\prime} v_{1} d x \\
& \left.\quad=-2 \lim _{\lambda \rightarrow 0} \frac{1}{\lambda^{2}} \int_{\Omega_{\lambda 1}}|u-v|\left[\frac{4 x_{1}^{2}}{\sqrt{1+x_{1}^{2}+x_{2}^{2}}}-2\right]\left(x_{2}-x_{1}^{2}-\lambda\right)\right) \nu_{1} d x \\
& \quad \leq 2 \lim _{\lambda \rightarrow 0} \frac{1}{\lambda} \int_{\Omega_{\lambda 1}}|u-v|\left[\frac{4 x_{1}^{2}}{\sqrt{1+x_{1}^{2}+x_{2}^{2}}}-2\right] v_{1} d x \\
& \quad=2 \int_{\Sigma_{1}}|u-v|\left[\frac{4 x_{1}^{2}}{\sqrt{1+x_{1}^{2}+x_{2}^{2}}}-2\right] v_{1} d \Sigma \\
& \quad=0 .
\end{aligned}
$$

Let $\lambda \rightarrow 0$ in (4.1). Then by $|f(\cdot, u)-f(\cdot, v)| \leq c|u-v|$, using (4.5)-(4.6), we have

$$
\begin{aligned}
& \int_{\Omega}|u(x, s)-v(x, s)| v_{1}(x) d x \\
& \quad \leq \int_{\Omega}|u(x, \tau)-v(x, \tau)| v_{1}(x) d x+c \int_{\tau}^{s} \int_{\Omega}|u-v| v_{1}(x) d x d t
\end{aligned}
$$

By the Gronwall lemma, we have

$$
\int_{\Omega}|u(x, s)-v(x, s)| v_{1}(x) d x \leq c^{*} \int_{\Omega}|u(x, \tau)-v(x, \tau)| v_{1}(x) d x
$$

where $c^{*}=e^{c T}$. 
Let $\tau \rightarrow 0$.

$$
\int_{\Omega}|u(x, s)-v(x, s)| v_{1}(x) d x \leq c^{*} \int_{\Omega}|u(x, 0)-v(x, 0)| v_{1}(x) d x .
$$

\section{Acknowledgements}

The author would like to thank BVOP for considering this paper to be published.

\section{Funding}

The paper is supported by Natural Science Foundation of Fujian province (2019J01858), supported by the Open Research Fund Program from Fujian Engineering and Research Center of Rural Sewage Treatment and Water Safety, and supported by SF of Xiamen University of Technology, China.

\section{Availability of data and materials}

Not applicable.

\section{Competing interests}

The author declares that he has no competing interests.

\section{Authors' contributions}

The author read and approved the final manuscript.

\section{Publisher's Note}

Springer Nature remains neutral with regard to jurisdictional claims in published maps and institutional affiliations.

\section{Received: 15 November 2018 Accepted: 10 May 2019 Published online: 21 May 2019}

\section{References}

1. Antonelli, F., Barucci, E., Mancino, M.E.: A comparison result for FBSDE with applications to decisions theory. Math. Methods Oper. Res. 54, 407-423 (2001)

2. Escobedo, M., Vazquez, J.L., Zuazua, E.: Entropy solutions for diffusion-convection equations with partial diffusivity. Trans. Am. Math. Soc. 343, 829-842 (1994)

3. Crandall, M.G., Ishii, H., Lions, P.L.: User's guide to viscosity solutions of second partial differential equations. Bull. Am. Math. Soc. (N.S.) 27, 1-67 (1992)

4. Antonelli, F., Pascucci, A.: On the viscosity solutions of a stochastic differential utility problem. J. Differ. Equ. 186, 69-87 (2002)

5. Citti, G., Pascucci, A., Polidoro, S.: Regularity properties of viscosity solutions of a non-Hormander degenerate equation. J. Math. Pures Appl. 80, 901-918 (2001)

6. Zhan, H.: The solution of the Cauchy problem for a quasilinear degenerate parabolic Eq. Chin. Anal. Math., Ser A. 33 449-460 (2012) (in Chinese)

7. Zhan, $\mathrm{H}$.: The study of the Cauchy problem of a second order quasilinear degenerate parabolic equation and the parallelism of a Riemannian manifold. Doctorial Thesis, Xiamen University (2004)

8. Zhan, H., Zhao, J.: The stability of solutions for second quasilinear degenerate parabolic equation. Acta Math. Sinica (Chin. Ser.) 50, 615-628 (2007)

9. Zhan, H.: Initial boundary value problems of an equation from mathematical finance. Chin. Anal. Math., Ser. B 37, 465-482 (2016)

10. Chen, G.Q., Perthame, B.: Well-posedness for non-isotropic degenerate parabolic-hyperbolic equations. Ann. Inst. Henri Poincaré, Anal. Non Linéaire 20, 645-668 (2003)

11. Chen, G.Q., DiBendetto, E.: Stability of entropy solutions to Cauchy problem for a class of nonlinear hyperbolic-parabolic equations. SIAM J. Math. Anal. 33, 751-762 (2001)

12. Karlsen, K.H., Risebro, N.H.: On the uniqueness of entropy solutions of nonlinear degenerate parabolic equations with rough coefficient. Discrete Contin. Dyn. Syst. 9, 1081-1104 (2003)

13. Bendahamane, M., Karlsen, K.H.: Renormalized entropy solutions for quasilinear anisotropic degenerate parabolic equations. SIAM J. Math. Anal. 36, 405-422 (2004)

14. Carrillo, J.: Entropy solutions for nonlinear degenerate problems. Arch. Ration. Mech. Anal. 147, 269-361 (1999)

15. $\mathrm{Wu}, \mathrm{Z}$., Zhao, J.: The first boundary value problem for quasilinear degenerate parabolic equations of second order in several variables. Chin. Anal. Math., Ser. B 4 319-358 (1983)

16. Krožkov, S.N.: First order quasilinear equations in several independent variables. Math. USSR Sb. 10, 217-243 (1970)

17. Kobayasi, K., Ohwa, H.: Homogeneous Dirichlet problems for quasilinear anisotropic degenerate parabolic-hyperbolic equations. J. Differ. Equ. 252, 4719-4741 (2012)

18. Lions, P.L., Perthame, B., Tadmor, E.: A kinetic formulation of multidimensional conservation laws and related equations. J. Am. Math. Soc. 7, 169-191 (1994)

19. Li, Y., Wang, Q.: Uniqueness and existence for anisotropic degenerate parabolic equations with boundary conditions on a bounded rectangle. J. Differ. Equ. 252, 137-167 (2012) 